COMMENTARY

\title{
Definition of an optimal strategy to evaluate and follow-up adrenal incidentalomas: time for further research
}

\author{
Massimo Terzolo, Giuseppe Reimondo and Alberto Angeli \\ Medicina Interna I, Dipartimento di Scienze Cliniche e Biologiche, Facoltà di Medicina e Chirurgia San Luigi Gonzaga, Università di Torino, \\ ASO San Luigi Gonzaga, Regione Gonzole, 10, 10043 Orbassano, Torino, Italy \\ (Correspondence should be addressed to M Terzolo; Email: terzolo@usa.net)
}

\begin{abstract}
Clinical endocrinologists have to deal frequently with adrenal incidentalomas in their practice. However, no comprehensive guidelines have been published by professional societies to guide assessment and management of adrenal incidentalomas and current recommendations are mainly based on the NIH state-of-the-science statement or expert opinions. An accompanying paper in this issue of the journal provides a critical revision of the relevant literature on this clinically important topic. The provocative conclusion of the authors is that current recommendations are burdened by high costs, little clinical benefit and also risk of inducing cancer. This paper has the merit to outline the limits of proposed protocols and to stimulate fruitful discussion; however, many aspects represent the personal view of the authors rather than evidence-based assumptions. The purpose of the present comment is to provide a more balanced view addressing the arguments that are condivisible and those that are based on inconsistent data. To add further to the argument of discussion, we also outline a clinically-oriented strategy for follow-up. In conclusion, the need for further research appears evident to define more efficient guidelines.
\end{abstract}

European Journal of Endocrinology $161529-532$

The serendipitous discovery of an adrenal tumour (adrenal incidentaloma) is a by-product of modern technology, and the routine use of sophisticated radiological techniques has revealed a problem that is not new but is increasingly recognized in current medical practice (1-3). Before the availability of sensitive imaging techniques many adrenal tumours remained undiagnosed throughout life, as demonstrated by their great prevalence in autopsy series (1).

The unexpected discovery of an adrenal mass is a challenge for the physician who needs to know the different management strategies of adrenal incidentalomas that vary according to their malignant potential and secretory status. In the era of evidence-based medicine, the process of clinical decision making should be guided by clear demonstration that a given adrenal tumour can cause harm to the patient while an effective treatment is available to limit attendant morbidity and mortality. Regretfully, for adrenal incidentalomas we only have low levels of evidence generated by case-control studies, case series, or expert opinions; consequently, the management of such tumours is largely empirical (4).

In this issue of the European Journal of Endocrinology, Cawood et al. (5) dealt with this difficult topic and the authors should be commended for having made a systematic revision of current strategies for initial evaluation and follow-up of adrenal incidentalomas. This is a difficult task since adrenal incidentaloma is not a uniform disease and relevant papers may be found in different speciality journals. In their work, Cawood et al. (5) tried to include studies whose results can be generalized to the practice of clinical endocrinologists and this is a specific merit of the paper, since frequency distribution of the adrenal tumours that may be detected incidentally depends heavily on referral $(1,6)$.

However, the process of literature search and selection of papers that are so heterogeneous as to study methodology, definition of incidentaloma and quality of data reporting is undoubtedly prone to a personal judgement that may not be entirely agreed on. As a matter of fact, it is understandable that series including patients with current or previous history of malignancy may be biased; however, patients with no evidence of cancer in whom an adrenal mass is detected for reasons other than oncological surveillance are represented in endocrine series (6). Following the same line, we agree that surgical series over-represent secreting or malignant tumours, but also some series recruited in endocrine centres have included patients with overtly hypersecreting tumours $(6,7)$. The bottom line is that the inclusion and exclusion criteria were inconsistently reported in published studies making it difficult to set predefined criteria to rule in or out 
potentially relevant studies. Moreover, Cawood et al. (5) argued that ultrasonography as the primary imaging modality may introduce a selection bias, but in many series published over the past decades that are included in their analysis, adrenal incidentalomas have been detected also by this technique (8-12). Notwithstanding some issues with literature scan, the paper by Cawood et al. (5) provides some important facts and figures, and some speculations as well, that may stimulate fruitful discussion.

First, the frequency of tumours that can be definitively dangerous for the patient, such as primary or secondary malignancies and hypersecreting tumours is low among patients with adrenal incidentalomas who are currently referred to endocrinologists. In their review, Cawood et al. (5) gave a median estimate around $2.0 \%$ for adrenocortical cancer, $<1.0 \%$ for adrenal metastases and around $3.0 \%$ for phaeochromocytoma. It has to be pointed out that these figures are lower than those generally reported in highlyreferenced reviews that did not used a narrow definition of adrenal incidentaloma but accepted all studies with their own definition (13-15).

Secondly, subclinical Cushing's syndrome is the most frequent hormonal disorder among adrenal incidentalomas with a median frequency of about $6.0 \%$ (5). Despite the frequency of this condition varying remarkably among the original studies, due to different diagnostic algorithms and criteria to qualify subclinical hypercortisolism, the finding that clinically inapparent adrenal adenomas may secrete cortisol autonomously, and possibly in some (slight) excess, is consistent throughout the literature (7). However, the clinical benefit associated with a diagnosis of subclinical Cushing's syndrome remains unsettled according to the present review (5).

Thirdly, the systematic follow-up of all adrenal incidentalomas considered to be benign and nonfunctional, by means of repeated CT and a number of endocrine tests, may carry little benefit and even cause a small increase in the risk of radiation-induced cancers. If the false-positive rate for functional or malignant lesions is typically five times greater than the true positive rate at the time of diagnosis, it becomes 50 times greater during follow-up (5). In the light of data presented, Cawood et al. (5) argued against a biochemical and imaging follow-up on a routine basis for the adrenal incidentalomas that were thought to be benign and non-secreting, or causing subclinical Cushing's syndrome. With the exception of tumours of indeterminate dignity, which may be submitted to a second CT after some months, the standard management should be that after the initial evaluation an adrenal incidentaloma is either removed surgically or discharged from follow-up (5).

This provocative conclusion is, in our opinion, the main contribution of the present paper to a topic that is of great relevance for clinical endocrinologists.
We believe, as the same authors acknowledge, that their estimates are objectionable for the inherent limitations of previous studies and methodology used to extract and process data from the literature; however, we share some arguments.

The authors are right when arguing that most adrenal incidentalomas are benign adrenocortical adenomas that likely account for $\sim 80 \%$ of all tumours also in our experience, even if a precise estimate is impossible because adrenocortical adenomas are rarely excised (4). Thus, it is reasonable that even a test with excellent operating characteristics will inevitably lead to a number of false-positive results for non-adenomatous tumours. Having said this, the discussion on costs generated by (false) positive testing should be tempered by an important clinical argument. An adrenal incidentaloma has a low pre-test probability of being an adrenocortical cancer or a phaeochromocytoma, but both these tumours are potentially lethal and can be cured, or at least patient's survival can be greatly improved, by timely adrenalectomy $(16,17)$. This justifies the use of high-sensitive diagnostic procedures and a low threshold for recommending surgery in doubtful cases.

We also agree with Cawood et al. (5) that subclinical Cushing's syndrome accounts for most 'functioning' tumours, when a strict definition of adrenal incidentaloma is used. However, subclinical Cushing's syndrome remains an ill-defined entity of questionable clinical importance $(6,7)$. We are sceptical about the authors' estimate of sensitivity and specificity of what is considered the best test, the overnight $1 \mathrm{mg}$ dexamethasone test (6). By definition, subclinical Cushing's syndrome applies to a state of deranged cortisol secretion without any specific sign of hypercortisolism and in the absence of any clear clinical correlate it is virtually impossible to define the rate of false positive or false negative results of any given test (7). Moreover, Cawood et al. (5) did not consider that several versions of the dexamethasone test were used (the $1 \mathrm{mg}$ overnight test, the $3 \mathrm{mg}$ overnight test, and the classic 2-day low-dose test) with variable cortisol cutpoints in the different studies $(18,19)$.

Cawood et al. (5) casted doubts on the value of extensive testing for subclinical Cushing's syndrome and, in principle, we may agree on this assumption. In our current practice, however, we are applying a more flexible approach guided by clinical judgement. We do not procede with further testing in patients suppressing cortisol below $50 \mathrm{nmol} / \mathrm{l}$ after $1 \mathrm{mg}$ overnight dexamethasone test but we may consider extending evaluation in patients with post-dexamethasone cortisol of $138 \mathrm{nmol} / \mathrm{l}$, or greater, when features of the metabolic syndrome or osteoporotic fractures are present. As a matter of fact, recent data have been published that suggest a higher than expected frequency of occult Cushing's syndrome in these two clinical scenarios $(20,21)$. Moreover, there is evidence 
that the slight amount of cortisol excess caused by clinically inapparent adrenocortical adenomas, even if insufficient to give a full-blown Cushingoid phenotype, may promote development of insulin resistance and its attendant clinical manifestations $(22,23)$. However, these studies suffer from the limit of a case-control design and do not prove definitively a cause and effect relationship.

Concerning the last, and most important, issue raised by Cawood et al. (5), they outlined correctly that a benign adrenal incidentaloma undergoes malignant transformation rarely, if ever (5). Moreover, the risk of developing clinically significant hormone hyperfunction during follow-up is also low (5). In this context, the risk of evolution from subclinical to clinically overt Cushing's syndrome, although depending upon the definition of the disorder, should not be a major concern (7). On the other hand, spontaneous normalization of subclinical hypercortisolism was also observed in some reports not included in the present analysis, raising the possibility that cortisol output by an adrenocortical adenoma may be variable over the course of time $(7,24)$. As a consequence, Cawood et al. (5) argued that follow-up of adrenal incidentalomas initially considered to be benign and not-functional is likely to result in significant costs, both financial and emotional, due to frequent false positive results, carries little clinical benefit and even confers a non-negligible risk of fatal cancer. Thus, they recommend against follow-up of all adrenal incidentalomas with repeated imaging and hormone work-up as a routine measure.

We consider that some arguments on the shortcomings of current follow-up recommendations are sound but also think that a more balanced view should be offered to the reader of the European Journal of Endocrinology.

In our opinion, patients who are not candidates for surgery (plausibly the majority) should be enrolled in a program of regular and careful clinical follow-up to detect, treat, and control central obesity, hypertension, glucose intolerance, dyslipidemia. Cardiovascular risk factors are over-represented in patients with adrenal incidentalomas, either because they are exposed to a chronic cortisol excess or for a referral bias (such patients are more likely to undergo imaging procedures); thus, advising either lifestyle changes or treatment with drugs that have been demonstrated to be protective for metabolic and cardiovascular complications should be an important task for clinical endocrinologists (25). Patients who develop clinical signs of hormone excess, or experience worsening of their metabolic status and cardiovascular risk profile despite optimal medical treatment should be re-evaluated for endocrine function. Concerning imaging, we recommend repeating the CT scan once after 3-6 months in all patients, to be sure of not missing a fast-growing tumour whose malignant potential was not recognized at first evaluation.
This event is exceptional in our practice, thus underlining that current imaging methodology, albeit imperfect, allows recognition of dangerous tumours (primary and secondary adrenal malignancies, phaeochromocytomas) in the vast majority of cases (4). Patients with small tumours, $<2 \mathrm{~cm}$, do not need further imaging in most cases, but for larger tumours the decision to proceed or not with imaging follow-up study should be tailored on the overall imaging phenotype of the mass, patient age, history and physical examination, and results of endocrine evaluation at baseline. The risk of cancer associated with CT radiation exposure is a matter of debate but the assessment of the risk by using the linear no-threshold extrapolation model, which assumes that cancer induction is proportional to dose even for the smallest doses, may overestimate the risk (26). If this is a concern, however, we may consider ultrasonography that can easily detect many tumours of at least $3 \mathrm{~cm}$, particularly on the right side, as a safe and cheap technique for follow-up (27). We acknowledge that this more clinically-oriented strategy is based purely on pragmatism, but has the merit of reducing costs and, possibly, increasing benefits compared to the current strategies. Moreover, it takes into account the fact that many patients are worried of no follow-up at all.

In summary, we share the conclusion of Cawood et al. (5) that it is time for a re-think on current recommendations for assessment and management of adrenal incidentalomas. However, we have to recognize that the natural history of these tumours remains virtually unknown. Thus, it is of the utmost importance to establish prospective studies with clearly defined entry criteria and standardized evaluation and management protocols to appraise the long-term outcome of such patients. At the same time, we urgently need to establish a gold standard for the diagnosis of subclinical Cushing's syndrome and to evaluate the impact of this diagnosis on patient's outcome. So, it is time for further (and better) research to attain evidence that should substantiate the implementation of future guidelines.

\section{Declaration of interest}

The authors declare that there is no conflict of interest that would prejudice the impartiality of this scientific work.

\section{Funding}

This research did not receive any specific grant from any funding agency in the public, commercial or not-for-profit sector.

\section{References}

1 Kloos RT, Gross MD, Francis IR, Korobkin M \& Shapiro B. Incidentally discovered adrenal masses. Endocrine Reviews 1995 $16460-484$. 
2 Aron DC. The adrenal incidentaloma: disease of modern technology and public health problem. Reviews in Endocrine and Metabolic Disorders 20012 335-342.

3 Young WF. Management approaches to adrenal incidentalomas: a view from Rochester, Minnesota. Endocrinology and Metabolism Clinics of North America 200029 159-185.

4 Terzolo M, Bovio S, Pia A, Reimondo G \& Angeli A. Management of adrenal incidentaloma. Best Practice and Research. Clinical Endocrinology E Metabolism 200923 233-243.

5 Cawood T, Hunt P, O'Shea D, Cole D \& Soule S. Recommended evaluation of adrenal incidentalomas is costly, has high false positive rates and confers a risk of fatal cancer that is similar to the risk of the adrenal lesion becoming malignant; time for a re-think? European Journal of Endocrinology 2009161 511-525.

6 Grumbach MM, Biller BM, Braunstein GD, Campbell KK, Carney JA, Godley PA, Harris EL, Lee JK, Oertel YC, Posner MC, Schlechte JA \& Wieand HS. Management of the clinically inapparent adrenal mass ('incidentaloma'). Annals of Internal Medicine 2003138 424-429.

7 Terzolo M, Bovio S, Reimondo G, Pia A, Osella G, Borretta G \& Angeli A. Subclinical Cushing's syndrome in adrenal incidentalomas. Endocrinology and Metabolism Clinics of North America 200534 423-439.

8 Bondanelli M, Campo M, Trasforini G, Ambrosio MR, Zatelli MC, Franceschetti P, Valentini A, Pansini R \& degli Uberti EC. Evaluation of hormonal function in a series of incidentally discovered adrenal masses. Metabolism 199746 107-113.

9 Barzon L, Scaroni C, Sonino N, Fallo F, Paoletta A \& Boscaro M. Risk factors and long-term follow-up of adrenal incidentalomas. Journal of Clinical Endocrinology and Metabolism 199984 520-526.

10 Mantero F, Terzolo M, Arnaldi G, Osella G, Masini AM, Ali A, Giovagnetti M, Opocher G \& Angeli A. A survey on adrenal incidentaloma in Italy. Study Group on Adrenal Tumors of the Italian Society of Endocrinology. Journal of Clinical Endocrinology and Metabolism 200085 637-644.

11 Favia G, Lumachi F, Basso S \& D’Amico DF. Management of incidentally discovered adrenal masses and risk of malignancy. Surgery $2000 \mathbf{1 2 8} 918-924$.

12 Libe R, Dall'Asta C, Barbetta L, Baccarelli A, Beck-Peccoz P \& Ambrosi B. Longterm follow-up study of patients with adrenal incidentalomas. European Journal of Endocrinology $2002 \mathbf{1 4 7}$ 489-494.

13 Mansmann G, Lau J, Balk E, Rothberg M, Miyachi Y \& Bornstein SR. The clinically inapparent adrenal mass: update in diagnosis and management. Endocrine Reviews 200425 309-340.

14 Barzon L, Sonino N, Fallo F, Palu G \& Boscaro M. Prevalence and natural history of adrenal incidentalomas. European Journal of Endocrinology $2003149273-285$.
15 Young WF. The incidentally discovered adrenal mass. New England Journal of Medicine $2007 \mathbf{3 5 6} 601-610$.

16 Allolio B \& Fassnacht M. Adrenocortical carcinoma: clinical update. Journal of Clinical Endocrinology and Metabolism $2006 \mathbf{9 1}$ 2027-2037.

17 Lenders JW, Eisenhofer G, Mannelli M \& Pacak K. Phaeochromocytoma. Lancet 2005366 665-675.

18 Lavoie H \& Lacroix A. Partially autonomous cortisol secretion by incidentally discovered adrenal adenomas. Trends in Endocrinology and Metabolism 19956 191-197.

19 Tsagarakis S, Vassiliadi D \& Thalassinos N. Endogenous subclinical hypercortisolism: diagnostic uncertainties and clinical implications. Journal of Endocrinological Investigation 2006 29 471-482.

20 Catargi B, Rigalleau V, Poussin A, Ronci-Chaix N, Bex V, Vergnot V, Gin H, Roger P \& Tabarin A. Occult Cushing's syndrome in type-2 diabetes. Journal of Clinical Endocrinology and Metabolism $2003 \mathbf{8 8}$ 434-441.

21 Chiodini I, Mascia ML, Muscarella S, Battista C, Minisola S, Arosio M, Santini SA, Guglielmi G, Carnevale V \& Scillitani A. Subclinical hypercortisolism among outpatients referred for osteoporosis. Annals of Internal Medicine $2007147541-548$.

22 Terzolo M, Pia A, Alì A, Osella G, Reimondo G, Bovio S, Daffara F, Procopio M, Paccotti P, Borretta G \& Angeli A. Adrenal incidentaloma: a new cause of the metabolic syndrome? Journal of Clinical Endocrinology and Metabolism $2002 \mathbf{8 7}$ 998-1003.

23 Tauchmanovà L, Rossi R, Biondi B, Pulcrano M, Nuzzo V, Palmieri EA, Fazio S \& Lombardi G. Patients with subclinical Cushing's syndrome due to adrenal adenoma have increased cardiovascular risk. Journal of Clinical Endocrinology and Metabolism $2002874872-4878$.

24 Bernini GP, Moretti A, Oriandini C, Bardini M, Taurino C \& Salvetti A. Long-term morphological and hormonal follow-up in a single unit on 115 patients with adrenal incidentalomas. British Journal of Cancer 200592 1104-1109.

25 Angeli A \& Terzolo M. Adrenal incidentaloma - a modern disease with old complications (editorial comment). Journal of Clinical Endocrinology and Metabolism 200287 4869-4871.

26 Tubiana M. Computed tomography and radiation exposure. New England Journal of Medicine 2008358850.

27 Fontana D, Porpiglia F, Destefanis P, Fiori C, Alì A, Terzolo M, Osella G \& Angeli A. What is the role of ultrasonography in the follow-up of adrenal incidentalomas? The Gruppo Piemontese Incidentalomi Surrenalici Urology $199954612-616$.

Received 9 June 2009

Accepted 17 August 2009 